cancer patients. Using WGS we aim to investigate further the heritable drivers of disease. Translation of genetic insights into better management of risk is pivotal to the value proposition for genomics, most likely through early cancer detection. In 2012 we began a surveillance study utilising whole body MRI. There are currently 76 participants and we have detected 14 new primary malignancies that have been treated with curative intent. We aim to inform identification of those at greatest heritable risk and influence clinical risk management in the genomic era of personalised medicine.

\section{DNA METHYLATION - IS IT READY FOR PRIME TIME IN GENETIC PATHOLOGY?}

\author{
Alexander Dobrovic \\ Translational Genomics and Epigenomics Laboratory, \\ University of Melbourne Department of Surgery, Austin \\ Hospital, Heidelberg, Vic, Australia
}

Assessing DNA methylation has not yet become commonplace in the diagnostic pathology laboratory. Our laboratory is one of the few laboratories in Australia that is NATA accredited for DNA methylation testing. Methylation analysis is more complex than mutational analysis as an appropriate region needs to identified and multiple $\mathrm{CpG}$ methylation sites within that region need to be analysed. It is also important to use quantitative methodologies. Interpretation can also be complex. Furthermore, consensus between laboratories can be difficult as discrepancies can occur due to different methodologies and different regions being analysed. Examples will be given of several genes for which methylation determination has clinical utility.

\section{INDICATIONS AND OUTCOMES OF RAPID TURN AROUND TIME WHOLE EXOME SEQUENCING STUDIES}

Michael F. Buckley ${ }^{1}$, George Elakis ${ }^{1}$, Sarah Lang ${ }^{1}$, Anna Richards $^{1}$, Corrina Cliffe ${ }^{1}$, Cheng-Yee Chan ${ }^{1, *}$, Edwin P. Kirk ${ }^{1}$, Ying Zhu ${ }^{1,2}$, Tony Roscioli ${ }^{1,3}$

${ }^{1}$ Randwick Genetics Laboratory, NSW Health Pathology, Randwick, Australia; ${ }^{2}$ Genetics of Learning Disability Service, Royal North Shore Hospital, St Leonards, Australia; and ${ }^{3}$ Neurosciences Research Australia, University of NSW, Randwick, Australia; *Current address: Canterbury Health Laboratories, Christchurch, New Zealand.

Rapid turn-around time whole exome sequencing has an established role in providing diagnoses for paediatric patients in intensive care units, with multiple publications from different clinical and laboratory services show-casing the clinical utility of that approach. The utility of rapid sequencing in other clinical settings has received comparatively less scrutiny. This report reviews the indications for referral and outcomes of rapid turnaround time whole exome studies requested for a range of clinical contexts from among cohort of 600 probands performed at a single laboratory centre over a period of 30 months.

Acknowledgement: The funding support of the Sydney Children's Hospital Network, NSW Health Pathology Genomics Directorate, and the Sydney Partnerships for Education, Research and Enterprise (SPHERE) is acknowledged with gratitude.

\section{EPIGENETIC CHANGES DURING TUMOUR DEVELOPMENT - FUNCTIONAL EPIGENETICS STUDY}

Clare Stirzaker $^{1,2}$, Ksenia Skvortsova $^{1}$,

Etienne Masle-Farquhar ${ }^{3}$, Christopher Goodnow ${ }^{2,3}$, Susan J. Clark ${ }^{1,2}$

${ }^{1}$ Epigenetics Research Laboratory, Genomics and Epigenetics Division, Garvan Institute of Medical Research, Sydney, Australia; ${ }^{2}$ St Vincent's Clinical School, UNSW Sydney, Sydney, Australia; and ${ }^{3}$ Immunology Division, Garvan Institute of Medical Research, Sydney, Australia

DNA methylation plays a key role in demarcation of regulatory regions, including promoter-associated $\mathrm{CpG}$ islands. While $\mathrm{CpG}$ islands are typically maintained in an unmethylated state, a proportion of islands are subject to hypermethylation during tumorigenesis. A key question is to understand what triggers the aberrant changes to the DNA methylation landscape in cancer. Using methylome sequencing, we have identified $\mathrm{CpG}$ islands that display partial DNA methylation encroachment across $5^{\prime}$ or $3^{\prime} \mathrm{CpG}$ island borders. CpG island methylation encroachment is a widespread phenomenon in clinical prostate and breast cancer and is commonly associated with gene suppression. By intergrating whole-genome DNA methylation sequencing and chromatin-modification sequencing data, we have revealed that the pattern of $\mathrm{H} 3 \mathrm{~K} 4$ monomethylated nucleosomes in normal cells is associated with the different modes of $\mathrm{CpG}$ island hypermethylation. Notably, we show that genetic manipulation of KMT2D results in concordant alterations in $\mathrm{H} 3 \mathrm{~K} 4 \mathrm{me} 1$ levels and corresponding $\mathrm{CpG}$ island border DNA methylation encroachment. Prostate cancer DNA harbouring KMT2D mutations also display reduced erosion of $\mathrm{CpG}$ island border methylation. Our findings establish a unique role for $\mathrm{H} 3 \mathrm{~K} 4 \mathrm{me} 1$ in the demarcation of $\mathrm{CpG}$ island borders in normal cells, which become eroded in cancer. ${ }^{1}$

\section{Reference}

1. Skvortsova K, Masle-Farquhar E, Luu PL, et al. DNA hypermethylation encroachment at $\mathrm{CpG}$ island borders in cancer is predisposed by H3K4 monomethylation patterns.Cancer Cell 2019; 35: 297-314.

\section{CANCER PHARMACOGENOMICS}

Munir Pirmohamed

MRC Centre for Drug Safety Science and Wolfson Centre for Personalised Medicine, The University of Liverpool, Liverpool, $U K$

About 1 in 2 to 1 in 3 people will develop cancer during their lifetime. Cancer is basically a genetic disease, and any patient who develops cancer has two genomes, the germline genome and the cancer genome. The cancer genome accumulates mutations, some of which may drive the progression of the disease (driver mutations). Our ability to sequence genomes has allowed (a) the development of targeted therapies (which target particular aberrant tumour proteins); (b) the identification of common genetic abnormalities across different tumour types leading to the development of tumour agnostic drugs; (c) the quantification of mutational and neoantigen burden so that novel predictive and interventional strategies can be developed; and (d) the development of liquid biopsy approaches for diagnosis, recurrence and prognosis. This is leading to transformational responses in 